\title{
DEVELOPMENT OF TECHNOLOGIES FOR HEALTHY AGING: SEARCHING FOR THE RIGHT WAY
}

\author{
A. Piau ${ }^{1,2,3}$,E. Campo ${ }^{2,3}$, B. Vellas ${ }^{1,4}$, F. Nourhashemi ${ }^{1,4}$
}

\begin{abstract}
Background: Medical imaging (e.g. PET-scan) and interventional (e.g. robotic surgery) technologies seem- to better fit the actual technological progress than do technologies for aging. The commercial market of aging care remains subdued since the introduction of low-technology devices decades ago (e.g. walker). Revisiting the evaluation and development methods of technologies to support healthy aging could help spread innovative technologies in this field. Methods and findings: In literature, a number of publications have been identified that addresses issues about technological devices that target the different needs of the older person. Nevertheless, a successful evaluation and development often remains unmet. This deficiency arises to a large extent from the confrontation of two worlds: that of technology which is not yet well versed in the field of healthy aging intervention, and the medical world which mainly uses the linear pharmaceutical drug development model. Many methods propose to tackle the global multidimensional evaluation of health technologies. However, they do not address the sequencing of the whole development and evaluation processes. In the present paper, we present a framework to help tackle the complexity of healthy aging technologies assessment and development. Conclusion: The evaluation and development methods usually adopted for healthy aging technologies are not appropriate and that all the collaborative multidisciplinary processes have to be revised.
\end{abstract}

Key words: Frailty, geriatrics, methodology, assessment, technologies.

\section{Introduction}

Although the adoption of technologies by older persons is still at an early stage, it provides great expectations. Technologies could promote healthy aging (1), independent living, comfort and safety no matter where the persons live. In nursing homes, technologies are potentially able to improve health care efficiency and quality, and also to protect resident privacy. They also potentially bypass the financial, geographical and organizational barriers restricting access to specific services (2). However, a number of obstacles appear to limit their dissemination (3), in surprising proportions. Several reasons can be reported. They include: the lack of objective assessment of these solutions, - inadequate plan for implementing them into practice and, in most cases, no real proof of efficacy is observed. Revisiting the evaluation and development methods could help spread innovative technologies in this field.

1. CHU de Toulouse, Gerontopôle, UPS, F-31400 Toulouse, France; 2. CNRS, LAAS, F-31400 Toulouse, France; 3. Univ de Toulouse, UT2J, LAAS, F-31100 Toulouse, France; 4. INSERM, UMR 1027, UPS, F-31400 Toulouse, France

Corresponding Author: A Piau, CHU de Toulouse, Gérontopole, Toulouse, France, piau.a@chu-toulouse.fr
The most documented studies are focused on telemedicine, telehealth and concern chronic illnesses (....). Limited evidence is available on the specific application of technologies dedicated to safety, autonomy or dependency prevention (4). Studies performed in this field rarely meet the usual standards of publications on health (4-8) Many of the trials carried out are too small and the endpoints are often inconsistent. To a large extent, this could arise from the confrontation of two different worlds: that of technology and its business community, which are not yet well versed in the field of health intervention, and that of the medical world, which mainly uses the linear pharmaceutical drug development model $(9,10)$.

Many authors call for trials which go beyond evaluation of clinical effectiveness (12-15). The point that these methods have in common is that they stress the multidimensional and iterative aspects of the assessement, the need of combining quantitative and qualitative criteria, and the importance of taking into account the setting in which the technology is implemented. Nevertheless, they do not address the sequencing of the development and evaluation processes. Law and Wason suggest adaptive approaches to trial design in telehealth studies (15), in which new decisions 
can be made about the design or progress of the trial, once the trial is already underway.

In the present brief commented review of literature, we propose a framework to help tackle the complexity of aging technologies development and assessment.

\section{Framework design}

\section{Issues to be taken into account}

Technical aspects are not only a matter of facing pure technological locks. Information feedback from medical, economic and ethical dimensions must be taken into account at each stage. This is particularly true for the man-machine interface where the end user's feedback is of primary importance. The medical dimension of evaluation concentrates on the efficacy and security of the technology on the subject. However, it also takes into account its value for public health, which concerns economic and societal dimensions. The difference between efficacy in a controlled context and efficacy in a real context, or effectiveness, seems to provide a greater impact - for technology, since it is highly dependent on the context in which it is deployed. The societal dimension assesses the global effect on society. Evaluation does not only concern a technical device but also its impact on overall management, as part of patient care. If alerts are automatically triggered from a technological device, the common global medical procedure should be well-known and practical implications should be assessed for all those who participate. Early involvement of all stakeholders is required to ensure technology implementation. To a very large extent, the legal and ethical dimensions cross other dimensions also, in particular medical (e.g. deontology) and societal (e.g. basic rights and responsibilities). As soon as the project specifications are decided, advice could be sought from a specialist on these issues and on the possible societal impact of technology. So, before designing a longitudinal evaluation, there is a preliminary question: does the use of the tool respect a legal framework (e.g. biomedical laws), or an ethical framework (e.g. ethics committee)? Should the device of interest be considered as a medical device? Is it necessary to perform the living lab evaluation in an accredited medical research environment? Furthermore, if technology generally carries low intrinsic medical risks, its use may lead to ethical consequences, e.g. geolocation in the case of a 3G-technology device. The economic dimension is a major asset in a highly restrictive economic environment. One of the barriers in implementing technology may be the lack of clear information as to who pays what and for whom.

\section{Phases of development and evaluation}

The process of technological development follows a series of phases that is less linear in comparison to that of drug development (see Box 1). We can distinguish a first phase, which corresponds to the phase of specifications. After the identification of a medical need (e.g. promoting healthy aging to prevent disability), the requirements are designed: identification of medical indicators (e.g. gait speed, weight), description of technological tools (e.g. gyroscope), and set up of a preliminary economic model. To better promote future implementation, this phase must involve all those involved in the evaluation, including end users. This could be considered as similar to the preclinical phase in the pharmaceutical process. The second phase corresponds to the technical "lab tests" of the initial solutions proposed. It validates the technical compliance of each "technological brick" in the laboratory with volunteer end users. This phase can be compared to phase I in drug development. The non-technical aspects will remain (Is the solution's cost economically acceptable? Are indicators of future adherence reported?). At the end of this phase, a first prototype is available. During the third phase, the prototype may be evaluated in a "living lab", validating clinical and technical feasibility according to the various medical scenarios established. The environment as well as the end user's attitude is new variables. Acceptability assessment allows iterative modifications before a wider dissemination. A comparison could be made here with phase II in drug development. A more extensive phase could be carried out. It would then match the phase III of drug development. This step could include technology distribution on a larger scale within a real-life context, thus enabling to evaluate the medical and social services rendered and the overall economic impact. All the variables are taken into account: the material and organizational context, the disability or the disease. A real appraisal can be made of the actual uses of the device. This evaluation is rarely carried out for reasons relevant to cost and time. Lastly, on the model of the "residual risk" evaluation that is carried out in post-marketing studies associated with a pharmacovigilance study, a final phase could be designed. It would use "observer" devices to follow-up the technology application in "real life" as part of observational studies. At each phase, information and feedback loops can be applied to modify the technology, the organization, or even the target of the application. This would be impossible in drug development, where per-protocol changes are prohibited for methodological reasons. Table 1 presents relationships between development phases and evaluation dimensions. 
Table 1

Relationships between development phases and evaluation dimensions



\section{Box 1}

\section{Characteristics of health technologies as compared to drug development}

- Technology evaluation is multidimensional and therefore multidisciplinary;

- As technology implies a change in overall strategy, rather than the introduction of a new tool, outcomes are reported to be more dependent to the technology's implementation context;

- Technology evaluation is non-linear, more iterative, flexible, pragmatic;

- Feedback loops are more effective in technology evaluation;

- So-called positivist quantitative research strategies need to be combined with constructivist qualitative strategies for technology evaluation;

- Less time is available for technology evaluation because of the rapidly changing market.

\section{Conclusion}

The evaluation and development methods usually adopted for aging technologies are not appropriate and the all-collaborative multidisciplinary processes have to be revised. Through this multidisciplinary and iterative approach, industrial partners can now be accompanied to develop relevant technologies in the field of healthy aging. It should be beneficial when setting up regional scalable platforms to develop and assess new devices both by academic and private partners. An example of this kind of platform is deployed by the Oregon Center for Aging and Technology at Oregon University. Today, the Toulouse Gérontopôle aims to achieve this approach in cooperation with industrial partners for the benefit of the aging population.

Author Contributions: A.P. and E.C. were involved in the writing of the manuscript's first draft and in the review of the subsequent drafts. B.V. and F.N. were involved in the conception of the article and reviewed the manuscript.

Sponsor's Role: no funding sources.

Disclosure statement: No potential conflicts of interest were disclosed.

\section{References}

1. Sparrow D, Gottlieb DJ, Demolles D, Fielding RA. Increases in muscle strength and balance using a resistance training program administered via a telecommunications system in older adults. Gerontol A Biol Sci Med Sci. 2011;66:1251-7.

2. Niefeld MR, Kasper JD. Access to ambulatory medical and long-term care services among elderly Medicare and Medicaid beneficiaries: organizational, financial, and geographic barriers. Med Care Res Rev 2005;62:300-19

3. Anderson J. Social, ethical, legal barriers to E-Health. Int J Med Inform 2007;76:480-3

4. Piau A, Campo E, Rumeau P, et al. Aging society and gerontechnology: a solution for an independent living? J Nutr Health Aging. 2014;18:97-112.

5. Martin S, Kelly G, Kernohan WG, et al. Smart home technologies for health and social care support. Cochrane Database Syst Rev 2008; 8:CD006412

6. Barlow J, Singh D, Bayer S, Curry R. A systematic review of the benefits of home telecare for frail elderly people and those with long-term conditions. J Telemed Telecare 2007;13:172-9 
7. Paré G, Moqadem K, Pineau G, St-Hilaire C. Clinical effects of home telemonitoring in the context of diabetes, asthma, heart failure and hypertension: a systematic review. J Med Internet Res 2010; 12: e21.

8. Whitten PS, Mair FS, Haycox A, et al. Systematic review of cost effectiveness studies of telemedicine interventions. BMJ 2002;324:1434-7.

9. Medical Research Council. A framework for development and evaluation of RCTs for complex interventions to improve health. [cited 2013 feb 11]. Available from: http://www.mrc.ac.uk/Utilities/Documentrecord/index. $\mathrm{htm}$ ?d=MRC003372.

10. Campbell NC, Murray E, Darbyshire J, et al. Designing and evaluating complex interventions to improve health care. BMJ 2007;334:455-9

11. Sorenson C, Drummond M, Borlum Kristensen F, Busse R. How can the impact of health technology assessments be enhanced? Policy Brief - WHO European Ministerial Conference on Health Systems. Health Systems and Policy Analysis, 2008
12. Granstrøm Ekeland A, Bowes A, Gammon D. Short version of MethoTelemed. Methodology to assess telemedicine applications. Norwegian Centre for Telemedicine, Norway, 2010. [cited 2012 dec 4]. Available from: http:/ / www.telemed.no/methotelemed.4565273-125741.html

13. Velasco-Garrido M, Busse R. Health technology assessment, An introduction to objectives, role of evidence, and structure in Europe. World Health Organization, on behalf of the European Observatory on Health Systems and Policies. Policy brief, 2005

14. Rialle V, Vuillerme N. Outline of a general framework for assessing e-health and gerontechnology applications: Axiological and diachronic dimensions. Gerontechnology 2010;9(2):245

15. Law LM, Wason JM. Design of telehealth trials - Introducing adaptive approaches. Int J Med Inform. 2014 Dec;83(12):870-880. 